\title{
The Origin of Galactic Cosmic Rays as Revealed by their Composition
}

\author{
Vincent Tatischeff, ${ }^{a, *}$ John C. Raymond, ${ }^{b}$ Jean Duprat, ${ }^{c}$ Stefano Gabici ${ }^{d}$ and Sarah \\ Recchia $^{e, a}$ \\ ${ }^{a}$ Université Paris-Saclay, CNRS/IN2P3, IJCLab, F-91405, Orsay, France \\ ${ }^{b}$ Center for Astrophysics | Harvard \& Smithsonian, 60 Garden St. Cambridge, MA 02138, USA \\ ${ }^{c}$ Institut de Minéralogie, de Physique des Matériaux et de Cosmochimie, CNRS-MNHN-Sorbonne \\ Université, F-75005, Paris, France \\ ${ }^{d}$ Université de Paris, CNRS, Astroparticule et Cosmologie, F-75006 Paris, France \\ ${ }^{e}$ Dipartimento di Fisica, Universitá di Torino \& INFN - Sezione di Torino, via Giuria 1, 10122 Torino, Italy \\ E-mail: vincent.tatischeff@csnsm.in2p3.fr
}

\begin{abstract}
Galactic cosmic-rays (GCRs) are thought to be accelerated in strong shocks induced by massive star winds and supernova explosions sweeping across the interstellar medium. But the phase of the interstellar medium from which the CRs are extracted has remained elusive up to now. We have studied in detail the GCR source composition deduced from recent measurements by the AMS-02, Voyager 1 and SuperTIGER experiments to obtain information on the composition, ionisation state and dust content of the GCR source reservoirs. The results of this data analysis suggest that the GCRs are mainly accelerated in superbubbles energised by the activity of massive star winds and supernova explosions. The resulting model explains well the measured abundances of all primary and mostly primary CRs from $\mathrm{H}$ to $\mathrm{Zr}$, including the overabundance of ${ }^{22} \mathrm{Ne}$.
\end{abstract}

$37^{\text {th }}$ International Cosmic Ray Conference (ICRC 2021)

July 12th - 23rd, 2021

Online - Berlin, Germany

\footnotetext{
${ }^{*}$ Presenter
} 


\section{Introduction}

Galactic cosmic rays (GCRs) are believed to be powered by supernova (SN) explosions in the interstellar medium (ISM). But the nature of the interstellar reservoir(s) from which the CRs are extracted is not well known. The full understanding of the origin of GCRs requires to be able to explain in detail the chemical composition of these particles. The most extensive work on this topic was done by Meyer et al. [1], who showed that refractory elements are relatively more abundant than volatile ones in the composition of GCRs at their sources, which can be explained if dust grains are injected into the diffusive shock acceleration process more efficiently than ions [2]. Meyer et al. [1] also found that among the GCR volatile elements, the heavier ones are enhanced relative to the lighter ones, which the authors attribute to a dependence of the acceleration efficiency on the magnetic rigidity of ions, and thus on their mass-to-charge ratio. Furthermore, as compared with the elemental composition of the solar system, the GCR source composition was found to be characterised by a general overabundance of heavier elements relative to $\mathrm{H}$ and $\mathrm{He}$. As discussed below (Section 2), this reported overabundance is related to the fact that CR protons and $\alpha$-particles have significantly different source spectra than the heavier elements [3-5], which was not known at the time of Ref. [1].

One of the most noticeable differences between the GCR source composition and the one of the solar system is the ${ }^{22} \mathrm{Ne} /{ }^{20} \mathrm{Ne}$ isotopic ratio, which is $\sim 5$ times higher in GCRs than in the Sun [6]. The measured overabundance of ${ }^{22} \mathrm{Ne}$ in the GCR composition suggests that these particles do not come solely from the average ISM, but that they contain a significant contribution from Wolf-Rayet (W.-R.) star winds enriched in helium-burning products [7]. But exactly how W.-R. wind material is incorporated into GCRs has been a matter of debate for decades [e.g. 6, 8-12].

In [13], we develop a model to analyse recent GCR abundances data with basic assumptions on the origin of these particles from the work of Ref. [1]. Here, we summarise our main results.

\section{Galactic cosmic ray abundances}

The GCR source abundances reported in the literature are usually normalisation factors of the injection spectra providing best fits to measured, propagated spectra [e.g. 16, and references therein]. This is justified as long as the CR data for different elements can be described with the same source spectrum. But data from Voyager 1 and AMS-02 have shown that CR protons and $\alpha$-particles have significantly different source spectra than the heavier elements [3, 4].

To estimate the source spectra and relative abundances of protons and $\alpha$-particles, we used the CR propagation model of Ref. [4], which provides a good fit to local interstellar spectra (LIS) measured by AMS-02. But to fit Voyager 1 data as well, we needed to introduce a low-energy break in the CR injection spectrum. Such a break can be expected in various scenarios of CR acceleration [3], but the reasons for the difference in the source spectra between $\mathrm{H}, \mathrm{He}$ and the heavier elements is not well understood.

The differential fluxes of GCR protons and $\alpha$ particles measured by Voyager 1 below $100 \mathrm{MeV}$ nucleon $^{-1}$ are significantly softer than those for the heavier nuclei, which implies that most of the GCR H and He ions are low-energy particles and that the total number of nuclei of each species injected from ISM reservoirs into the acceleration process depends on the minimum 


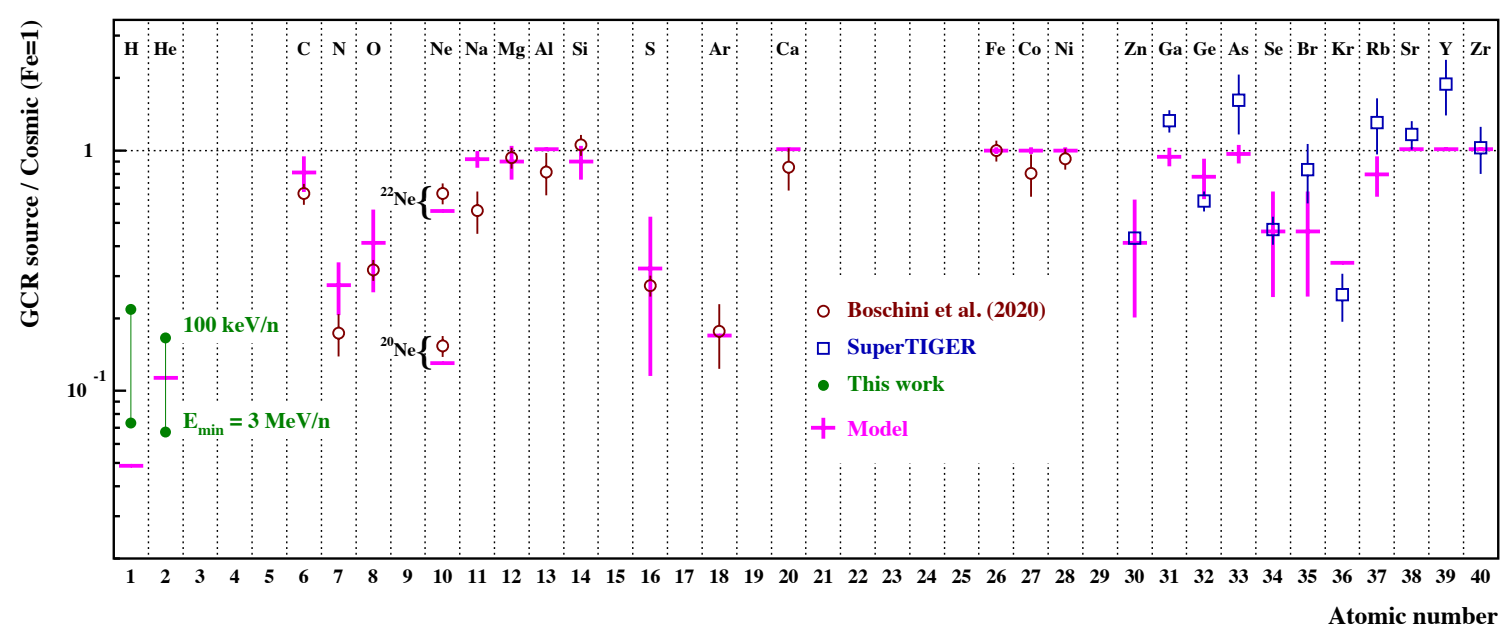

Figure 1: GCR source abundances relative to the standard cosmic (SC) composition of the ISM [14, 15]. The GCR abundances of $\mathrm{H}$ and He calculated in Ref. [13] are shown for two values of the CR minimum energy: $E_{\min }=0.1$ and $3 \mathrm{MeV}$ nucleon $^{-1}$. The GCR source abundances of the heavier elements are taken from Boschini et al. [16, 17] and SuperTIGER data [18]. The abundances of ${ }^{20} \mathrm{Ne}$ and ${ }^{22} \mathrm{Ne}$ are shown separately. The measured abundances are compared to those predicted in the best-fit model of Ref. [13], where the GCR volatiles are produced in superbubbles (SBs) and the ${ }^{22} \mathrm{Ne}$-rich component is accelerated in wind termination shocks (WTSs). Error bars on the model points take into account the uncertainties on the element fraction in ISM dust.

CR energy, $E_{\min }$. Calculated GCR abundances of $\mathrm{H}$ and He are shown in Figure 1 for two values of $E_{\min }$. We see that the abundances of these two species relative to the SC composition are similar to those of the other volatile elements $\mathrm{N}, \mathrm{Ne}$ and Ar, provided that $E_{\min }$ is of the order of a few hundred $\mathrm{keV}_{\text {nucleon }}{ }^{-1}$. The results of the model shown in this Figure are presented below.

\section{Galactic cosmic ray composition model}

We aim at explaining the source composition of GCRs from $\mathrm{H}$ to $\mathrm{Zr}$ with three basic assumptions. First, the overabundance of refractory elements in the GCR composition results from efficient acceleration of ISM dust grains in strong shocks, as studied in detail by [2]. Assuming that the measured GCR abundance of any element $i$ comes from two sources, one from a gas reservoir and another associated with ISM dust, i.e. $C_{\text {mes }}(i)=C_{\text {gas }}(i)+C_{\text {dust }}(i)$, the dust contribution can be written as:

$$
C_{\text {dust }}(i)=\operatorname{SC}(i) f_{d}(i) \epsilon_{\text {dust }},
$$

where $\mathrm{SC}(i)$ is the present-day stand cosmic (SC) abundance of element $i$ in the ISM [14, 15], $f_{d}(i)$ is the fraction of its abundance arising from dust, and $\epsilon_{\text {dust }}$ the efficiency of injection of refractory elements contained in ISM grains into the GCR population. The latter quantity depends on the evolution of shock waves in the ISM, the ambient medium density, the grain size distribution, but not of the mass and atomic number of the ions sputtered from the grains (i.e. it is independent of $i$ ).

Second, we assume that the reservoir of volatile elements from which GCRs are produced is enriched in W.-R. star wind material in which He-burning products are expelled during the WC and 
WO phases $[1,7,10]$. This is required to explain the high ${ }^{22} \mathrm{Ne} /{ }^{20} \mathrm{Ne}$ isotopic ratio measured in GCRs $[6,16]$. Assuming that the abundance of volatile elements in the GCR results from two dictinct sources, one with a SC composition and the other from W.-R. star winds $\left(C_{\mathrm{gas}}(i)=C_{\mathrm{gas}}^{\mathrm{SC}}(i)+C_{\mathrm{gas}}^{w}(i)\right)$ we can write

$$
C_{\mathrm{gas}}^{w}(i) \propto f_{w}(i) \operatorname{SC}(i)\left(1-f_{d}(i)\right)
$$

with $f_{w}(i)$ the enhancement of element $i$ in the W.-R. wind reservoir compared to the SC composition. In Ref. [13], we study two different scenarios for the overabundance of GCR ${ }^{22} \mathrm{Ne}$. The first one assumes that SN shocks in superbubbles (SBs) propagate in a medium enriched by W.-R. winds from the most massive stars of the parent $\mathrm{OB}$ associations [6, 9, 11]. The second scenario considers that stellar winds in massive star clusters are efficiently accelerated by wind termination shocks [WTSs; 12].

The third and last assumption is based on 2D PIC simulations of CR acceleration in collisionless shocks $[19,20]$, which show that the injection efficiency of ions into the diffusive shock acceleration process depends linearly on the magnetic rigidity of the particles and thus on the ion mass-to-charge ratio. The GCR abundance arising from the gas reservoir can then be written as:

$$
C_{\mathrm{gas}}(i)=\mathrm{SC}(i)\left(1-f_{d}(i)\right) \epsilon_{\mathrm{gas}}\left[x_{w} f_{w}(i) f_{A / Q}^{w}(i)+\left(1-x_{w}\right) f_{A / Q}^{\mathrm{SC}}(i)\right],
$$

where $\epsilon_{\text {gas }}$ is a global efficiency factor for the injection of interstellar ions into the GCR population, $x_{w}$ the contribution of the W.-R. wind reservoir to the GCR source gas population (in total number of atoms) and $f_{A / Q}^{j}(i)=\left(1-X_{0, i}^{j}\right) A_{i} / Q_{i}^{j}$, with $X_{0, i}^{j}, A_{i}$ and $Q_{i}^{j}$ the fraction of neutral atoms, the atomic mass and the mean ionic charge without the neutral fraction, for ions $i$ in the region immediately upstream of shock waves propagating in the medium of composition $j$ (SC or W.-R. winds). The gas reservoir of SC composition may include several phases of the ISM, such as a mixture of hot gas, warm neutral medium (WNM) and warm ionised medium (WIM). In this case, the mass-to-charge ratio factor in the above equation is obtained from

$$
f_{A / Q}^{\mathrm{SC}}(i)=\sum_{k} a_{k} f_{A / Q}^{\mathrm{SC}, k}(i)
$$

where $a_{k}$ is the relative contribution of the ISM phase $k$ to the GCR volatile production and $f_{A / Q}^{\mathrm{SC}, k}(i)$ the mass-to-charge ratio in the photoionisation precursor of shocks propagating in the $k$-phase.

Equations 1 and 3 can be easily combined to estimate the relative contributions of the ISM dust and gas reservoirs in the measured GCR abundances, as well as the relative contribution of the gas reservoir of SC composition. In [13], we carefully estimate the quantities $f_{d}, x_{w}, f_{w}, f_{A / Q}^{\mathrm{SC}}$ and $f_{A / Q}^{w}$ from various available data, and then derive $\epsilon=\epsilon_{\text {dust }} / \epsilon_{\text {gas }}$, as well as constraints on the GCR source reservoirs, from a fit of the model to the GCR abundance data.

\section{Results of the data analysis}

We considered five models covering the main assumptions for the origin of the GCR gas reservoir. In Model 1, we assume that the GCR volatiles of SC composition are extracted from SN remnants (SNRs) randomly distributed in the warm ISM, with $70 \%$ in the WNM and $30 \%$ in the WIM. We further assume that the high ${ }^{22} \mathrm{Ne} /{ }^{20} \mathrm{Ne}$ ratio of the GCR composition is due to the 

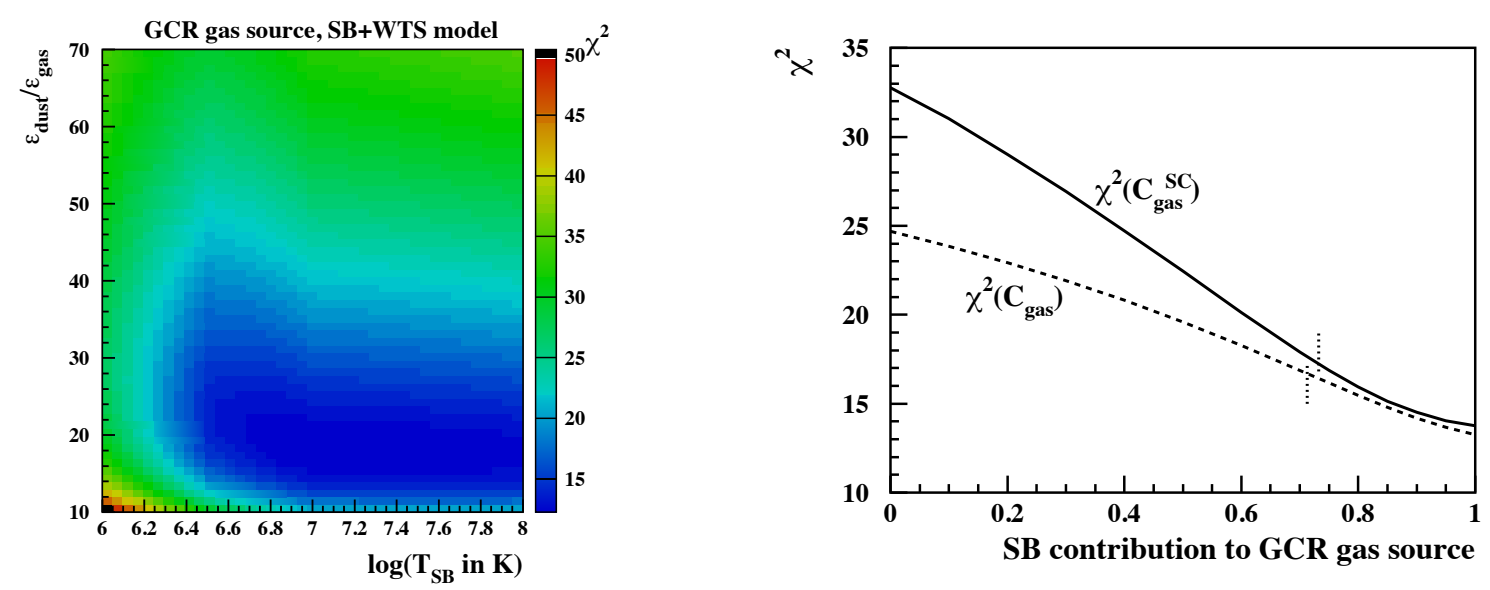

Figure 2: Left: Chi-square map from a fit of the GCR abundances from acceleration of ions in gas phase as a function of two free parameters, the SB temperature and the relative efficiency $\epsilon=\epsilon_{\mathrm{dust}} / \epsilon_{\mathrm{gas}}$, for the best-fit model (Model 3), where the GCR volatiles are accelerated in SBs and the ${ }^{22} \mathrm{Ne}$-rich component is produced in WTSs. Right: Chi-square values as a function of the SB contribution to the GCR gas source $\left(a_{\mathrm{SB}}\right.$; see Eq. 4), in a model where the reservoir of SC composition is a mixture of WNM, WIM and SB gas, and the ${ }^{22} \mathrm{Ne}$-rich component is produced in WTSs. The dashed and solid lines are for the normalised GCR data for the total gas source and for the gas source of SC composition, respectively [see 13]. The two vertical, dotted line segments at $a_{\mathrm{SB}} \sim 0.72$ mark the $1 \sigma$ limits for a fit with three free parameters at $\chi_{\min }^{2}+3.5$.

acceleration of ${ }^{22} \mathrm{Ne}$-rich stellar winds in WTSs. In Model 2, the GCR volatiles are accelerated in SBs. In this model, the high ${ }^{22} \mathrm{Ne}$ abundance in GCRs is explained by the enrichment of SB cores by the wind material of very massive stars before the first SN explosions. Model 3 also assumes that the GCR volatiles are produced in SBs, but here the ambient medium swept up by SN blast waves is supposed to be not significantly enriched in ${ }^{22} \mathrm{Ne}$. Instead, the Ne isotopic ratio at the GCR source is explained by the acceleration of massive star winds in WTSs. In Model 4, we assume that all SNRs in the ISM contribute equally to the production of the GCR volatiles, whose source reservoir is a mixture of $60 \% \mathrm{SB}$ material, $28 \% \mathrm{WNM}$ and $12 \% \mathrm{WIM}$. The ${ }^{22} \mathrm{Ne}$-rich component in this model is taken to be accelerated in SB cores enriched by massive star wind material. Finally, Model 5 is similar to Model 4, except that the high abundance of GCR ${ }^{22} \mathrm{Ne}$ is due to a contribution from particle acceleration in WTSs.

The best-fit results are obtained with Model 3. We obtained a reduced $\chi^{2}$ of 0.51 for this model, compared to $\chi_{v}^{2}=1.0,1.3,1.3$, and 0.70 for Models 1, 2, 4, and 5, respectively. The left panel of Figure 2 shows a chi-square map obtained when the SB temperature, $T_{\mathrm{SB}}$, is left as a free parameter. The temperature of the ambient medium determines the ionisation state of the elements in the preshock region, and thus their mass-to-charge ratio $\left(f_{A / Q}^{\mathrm{SC}}\right.$ in Eq. 3$)$. We derived a lower limit of $\log \left(T_{\mathrm{SB}}\right)>6.45\left(T_{\mathrm{SB}}>2.8 \times 10^{6} \mathrm{~K}\right)$, which is consistent with the measured temperature in the cores of SBs. As illustrated in the right panel of Figure 2, we found that SNRs in SBs contribute to the GCR volatile composition for more than 70\%. In comparison, using the observed distribution of young SNRs in our Galaxy and other observational guidance from ISM tracers, we estimated in [13] that $60 \%$ of all Galactic SNe explode in SBs, $28 \%$ in the WNM and 12\% in the WIM.

We see in Figure 3 that in Model 3, the normalised GCR abundance data for the dust and 

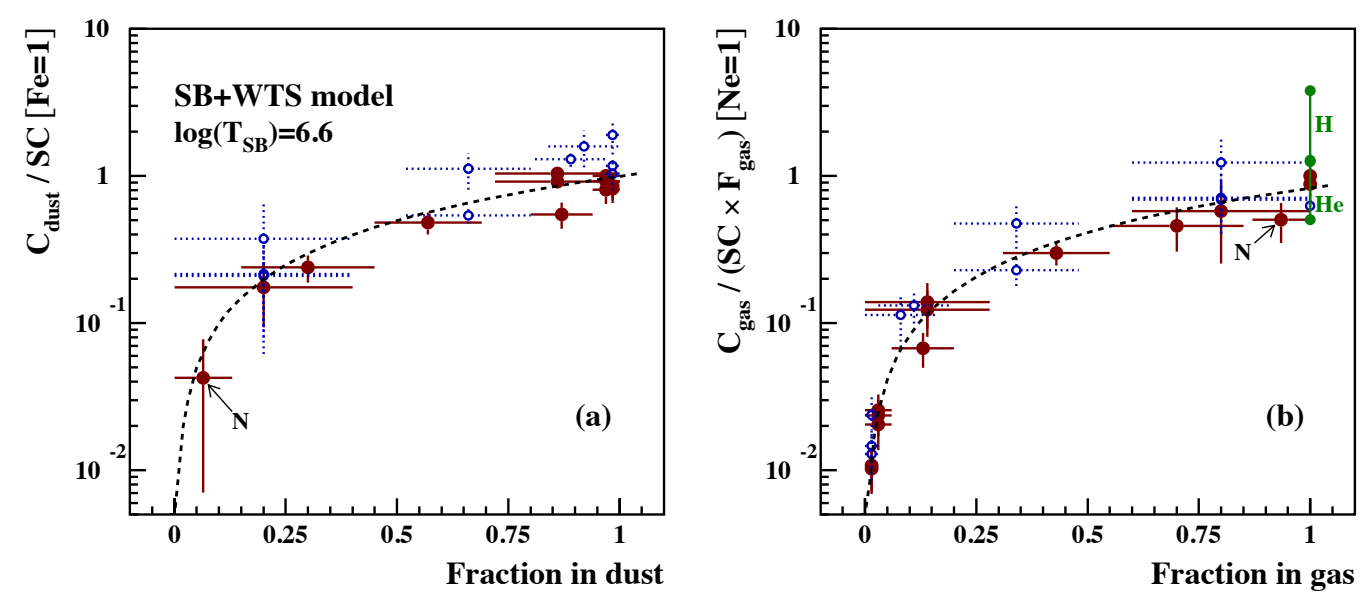

Figure 3: Normalised GCR abundance data for the dust $(a)$ and gas $(b)$ sources, as a function of the element fractions in ISM dust and gas, respectively. In the best-fit solution, the plotted quantities are expected to be proportional to $f_{d}$ and $f_{g}=1-f_{d}$, respectively (see Eqs. 1 and 3 ; here $F_{\text {gas }}=x_{w} f_{w} f_{A / Q}^{w}+\left(1-x_{w}\right) f_{A / Q}^{\mathrm{SC}}$ ); the dashed curves show best linear fits to the data. The data points are obtained from Model 3 with $\log \left(T_{\mathrm{SB}}\right)=6.6$ and $\epsilon=20.2$ - red filled circles: data from [16, 17], blue open circles: data from [18]. The $\mathrm{H}$ and $\mathrm{He}$ data in panel $(b)$ are represented by two connected points obtained for the minimum GCR source energy $E_{\min }=0.1$ and $3 \mathrm{MeV}$ nucleon $^{-1}$ (upper and lower points, respectively).

gas sources are well proportional to the element fractions in ISM dust and gas, respectively. In particular, the $\mathrm{N}$ abundance is in line with the general trend, which is not the case for Models 2 and 4 [see 13], where the high abundance of ${ }^{22} \mathrm{Ne}$ in GCRs is assumed to result from the enrichment of SB cores by the wind material of very massive stars. In these models, the SB cores are strongly enriched in ${ }^{14} \mathrm{~N}$ expelled by massive stars before their W.-R. WC and WO phases, and the N/Ne ratio is too high compared to the GCR data. The high abundances of GCR protons and $\alpha$ particles relative to heavy elements also favour an origin of these particles in the hot ISM, where the $A / Q$ selection effect is less important (however, see [21]).

\section{Conclusions}

The main conclusions of this work are the following:

- The reported overabundance of $\mathrm{C}$ and heavier elements in the GCR composition relative to $\mathrm{H}$ and $\mathrm{He}$ [e.g., 1] can be explained by the fact that CR protons and $\alpha$-particles have significantly different source spectra than the other elements. With a minimum CR source energy of the order of a few hundred $\mathrm{keV}$ nucleon ${ }^{-1}$, the GCR source abundances of $\mathrm{H}$ and He relative to the SC composition are similar to those of the other volatile elements $\mathrm{N}, \mathrm{Ne}$ and $\mathrm{Ar}$.

- The CR volatiles are mostly accelerated in Galactic SBs, from SNR shocks sweeping up a plasma of temperature $\gtrsim 2 \times 10^{6} \mathrm{~K}$. SNRs in the warm ISM contribute to the GCR volatile composition for less than $28 \%$ ( $1 \sigma$ limit), whereas about $40 \%$ of Galactic SNe occur in this phase and not in SBs. 
- The overabundance of ${ }^{22} \mathrm{Ne}$ in GCRs is most likely due to a relativeley small contribution of particle acceleration in WTSs of massive stars.

- From the CR-related $\gamma$-ray luminosity of the Milky Way, we estimated in [13] that the particle acceleration efficiency in both SN shocks and WTSs is of the order of $10^{-5}$, which is consistent with the prediction of the diffusive shock acceleration theory for strong shocks.

- The GCR refractory elements most likely originate from the acceleration and sputtering of dust grains in SNR shocks. We suggest that the GCR refractories could also be produced in SBs, if dust is continuously replenished in the SB interior through thermal evaporation of embedded molecular clouds swept up by SN shocks.

\section{Acknowledgements}

SG and VT acknowledge support from Agence Nationale de la Recherche (grant ANR-17CE31-0014). The work of SR is supported by the DePARTMENTS of ExCELLENCE grant awarded by the Italian Ministry of Education, the Research grant The Dark Universe: A Synergic Multimessenger Approach, No. 2017X7X85K funded by the MiUR and the Research grant TAsP funded by INFN.

\section{References}

[1] J.-P. Meyer, L.O. Drury and D.C. Ellison, Galactic Cosmic Rays from Supernova Remnants. I. A Cosmic-Ray Composition Controlled by Volatility and Mass-to-Charge Ratio, ApJ 487 (1997) 182 [astro-ph/9704267].

[2] D.C. Ellison, L.O. Drury and J.-P. Meyer, Galactic Cosmic Rays from Supernova Remnants. II. Shock Acceleration of Gas and Dust, ApJ 487 (1997) 197 [astro-ph/9704293].

[3] V. Tatischeff and S. Gabici, Particle Acceleration by Supernova Shocks and Spallogenic Nucleosynthesis of Light Elements, Annual Review of Nuclear and Particle Science 68 (2018) 377 [1803.01794].

[4] C. Evoli, R. Aloisio and P. Blasi, Galactic cosmic rays after the AMS-02 observations, Phys. Rev. D 99 (2019) 103023 [1904 . 10220].

[5] B. Schroer, C. Evoli and P. Blasi, Intermediate-mass and heavy Galactic cosmic-ray nuclei: The case of new AMS-02 measurements, Phys. Rev. D 103 (2021) 123010 [2102 . 12576].

[6] W.R. Binns, M.E. Wiedenbeck, M. Arnould, A.C. Cummings, J.S. George, S. Goriely et al., Cosmic-Ray Neon, Wolf-Rayet Stars, and the Superbubble Origin of Galactic Cosmic Rays, ApJ 634 (2005) 351 [astro-ph/0508398].

[7] M. Casse and J.A. Paul, On the stellar origin of the Ne-22 excess in cosmic rays, ApJ 258 (1982) 860.

[8] A. Maeder, Evolution of Chemical Abundances in Massive Stars - Part Two - Abundance Anomalies in Wolf-Rayet Stars in Relation with Cosmic-Rays and 22NE in Meteorites, A\&A 120 (1983) 130. 
[9] J.C. Higdon and R.E. Lingenfelter, The Superbubble Origin of ${ }^{22}$ Ne in Cosmic Rays, ApJ 590 (2003) 822.

[10] N. Prantzos, On the origin and composition of Galactic cosmic rays, A\&A 538 (2012) A80 [1112.4343].

[11] R.E. Lingenfelter, The Origin of Cosmic Rays: How Their Composition Defines Their Sources and Sites and the Processes of Their Mixing, Injection, and Acceleration, ApJS 245 (2019) 30 [1903.06330].

[12] S. Gupta, B.B. Nath, P. Sharma and D. Eichler, Realistic modelling of wind and supernovae shocks in star clusters: addressing ${ }^{22} \mathrm{Ne}{ }^{20} \mathrm{Ne}$ and other problems in Galactic cosmic rays, MNRAS 493 (2020) 3159 [1910 . 10168].

[13] V. Tatischeff, J.C. Raymond, J. Duprat, S. Gabici and S. Recchia, The Origin of Galactic Cosmic Rays as Revealed by their Composition, arXiv e-prints (2021) arXiv:2106.15581 [2106.15581].

[14] M.F. Nieva and N. Przybilla, Present-day cosmic abundances. A comprehensive study of nearby early B-type stars and implications for stellar and Galactic evolution and interstellar dust models, A\&A 539 (2012) A143 [1203. 5787].

[15] K. Lodders, H. Palme and H.P. Gail, Abundances of the Elements in the Solar System, Landolt B\&ouml;rnstein 4B (2009) 712 [0901.1149].

[16] M.J. Boschini, S. Della Torre, M. Gervasi, D. Grandi, G. Jóhannesson, G. La Vacca et al., Inference of the Local Interstellar Spectra of Cosmic-Ray Nuclei $Z \leq 28$ with the GALPROP-HELMOD Framework, ApJS 250 (2020) 27 [2006. 01337].

[17] M.J. Boschini, S. Della Torre, M. Gervasi, D. Grandi, G. Jóhannesson, G. La Vacca et al., The Discovery of a Low-energy Excess in Cosmic-Ray Iron: Evidence of the Past Supernova Activity in the Local Bubble, ApJ 913 (2021) 5 [2101. 12735].

[18] R.P. Murphy, M. Sasaki, W.R. Binns, T.J. Brandt, T. Hams, M.H. Israel et al., Galactic Cosmic Ray Origins and OB Associations: Evidence from SuperTIGER Observations of Elements ${ }_{26}$ Fe through ${ }_{40} \mathrm{Zr}$, ApJ 831 (2016) 148 [1608.08183].

[19] D. Caprioli, D.T. Yi and A. Spitkovsky, Chemical Enhancements in Shock-Accelerated Particles: Ab initio Simulations, Phys. Rev. Lett. 119 (2017) 171101 [1704.08252].

[20] A. Hanusch, T.V. Liseykina and M. Malkov, Acceleration of Cosmic Rays in Supernova Shocks: Elemental Selectivity of the Injection Mechanism, ApJ 872 (2019) 108 [1803.00428].

[21] B. Eichmann and J.P. Rachen, Nonthermal element abundances at astrophysical shocks, J. Cosmology Astropart. Phys. 2021 (2021) 049 [2010. 06523]. 